\title{
Traffic Based Optimization of Spectrum Sensing in Cognitive Radio Networks
}

\author{
Changhua Yao, ${ }^{1}$ Qihui $\mathrm{Wu},{ }^{1}$ and Linfang $\mathrm{Zhou}^{2}$ \\ ${ }^{1}$ PLA University of Science and Technology, Nanjing, China \\ ${ }^{2}$ PLA 73122 Army, Fuzhou, China \\ Correspondence should be addressed to Changhua Yao; ych2347@163.com
}

Received 21 April 2014; Revised 16 August 2014; Accepted 16 August 2014; Published 25 September 2014

Academic Editor: Masoud Hajarian

Copyright (c) 2014 Changhua Yao et al. This is an open access article distributed under the Creative Commons Attribution License, which permits unrestricted use, distribution, and reproduction in any medium, provided the original work is properly cited.

\begin{abstract}
We propose a more practical spectrum sensing optimization problem in cognitive radio networks (CRN), by considering the data traffic of second user (SU). Compared with most existing work, we do not assume that SU always has packets to transmit; instead, we use the actual data transmitted per second rather than the channel capacity as the achievable throughput, to reformulate the Sensing-Throughput Tradeoff problem. We mathematically analyze the problem of optimal sensing time to maximize the achievable throughput, based on the data traffic of SU. Our model is more general because the traditional Sensing-Throughput Tradeoff model can be seen as a special case of our model. We also prove that the throughput is a concave function of sensing time and there is only one optimal sensing time value which is determined by the data traffic. Simulation results show that the proposed approach outperforms existing methods.
\end{abstract}

\section{Introduction}

Cognitive radio (CR) is a promising technology to exploit the unused spectrum bands licensed to the primary user (PU) [1]. When the PU is inactive, the SU can transmit data on the idle channel; otherwise, SU have to keep silent. To provide enough protection of $\mathrm{PU}$, the secondary users are required to sense the channel. Therefore, spectrum sensing is of significant importance in CRN.

There is a fundamental problem called SensingThroughput Tradeoff which has been studied in depth [2-4]. From the SU's perspective, when the sensing time is longer, the probability of false alarm will be lower, and then there are more chances to transmit data which means the throughput is higher. However, the longer sensing time also shortens the allowable data transmission time of the secondary users. Hence, it is an important problem to find the optimal sensing time that maximizes the SU's throughput while providing adequate protection to the PU.

Some important results have been given in related references. References [2-4] study the tradeoff to get the optimal throughput. This problem is further studied under limited time constraints in [5]. The authors in [6] consider the effect of primary user traffic. References $[7,8]$ study the tradeoff problem considering the reporting overhead. The authors in [9] propose a discontinuous spectrum sensing scheme under energy-constrained environment and achieve remarkable energy savings with negligible throughput loss.

Though this problem has been extensively studied with significant results, to the best of our knowledge, existing researches all make an impractical assumption that SU has countless data packets to transmit all the time. Then the optimal sensing time problem is equivalent to the following problem: how to maximize the idle channel capacity in a frame time.

However, this assumption does not always hold because the amount of data packets depends on the traffic flow in practical systems. Actually, it is certainly not SU has countless packets to transmit all the time in practical scenarios. In methodology, the above assumption simplifies the sensing time optimization problem, because the data traffic would have a great influence on the optimal sensing time. It should be noted that the ultimate purpose of all the study on spectrum sensing and access is to complete the data traffic of SU. 


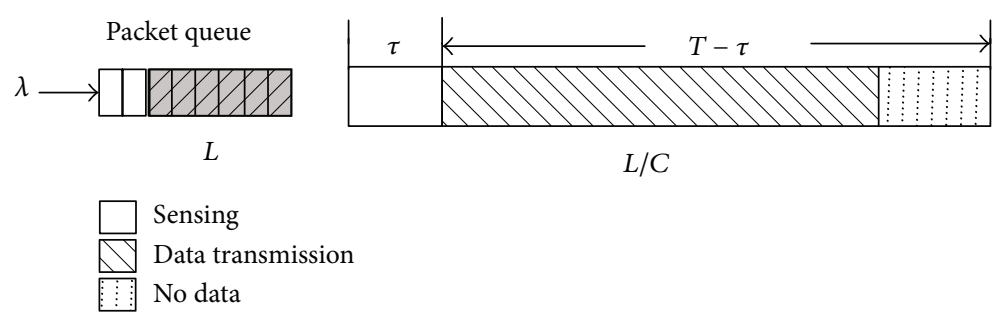

FIgURE 1: Frame structure.

In this paper, we discard the impractical assumption that SU has data packets to transmit all the time. We define the SU throughput as the actually transmitted data in the actual time spent, rather than the channel capacity in the frame time and reformulate the Sensing-Throughput Tradeoff problem based on the data traffic of SU. We propose a more general model and prove that the throughput is a concave function of sensing time and there is only one optimal sensing time value which is determined by the data traffic.

The rest of this paper is organized as follows. The system model is described in Section 2. In Section 3, the performance analysis is presented. Computer simulation results are provided in Section 4. Finally, we draw a conclusion of our work in Section 5.

\section{System Model}

2.1. Frame Structure. We consider a slotted frame structure like in [2]. We also assume that the PU is either absent or present during each frame duration. The slot time is $T$ which is divided into sensing duration $\tau$ and data transmission duration $T-\tau$, as shown in Figure 1. SU carries out spectrum sensing in the sensing duration and starts data transmission if $\mathrm{PU}$ is inactive by the sensing result. We denote that the channel capacity is $C$.

We, specially, discard the impractical assumption that SU has data packets to transmit all the time and focus on the actual SU throughput defined by transmitted data in the actual time spent, rather than the channel capacity obtained. In Figure 1, the data packet queue of SU is not always full, which is decided by the input traffic (actual demand). The SU may not always transmit data packets during the left time of slot $T-\tau$, the real data transmission time will be $L / C$, and then the actual throughput would be totally different with existing works where the assumption of countless data is adopted.

Obviously, our model is more practical because it is certainly not SU has countless packets to transmit all the time in practical scenarios. Actually, our model is more general compared to the existing one. The traditional model in [2] is a special case of our model when the data queue of SU is long enough for transmission duration $T-\tau$; that is, $L \geq(T-\tau) C$.

2.2. Definition of Throughput. In existing works, under the assumption that all the SUs have countless data packets to transmit all the time, the throughput would be computed by

$$
R_{1}=\frac{(T-\tau)}{T} \times C
$$

where $C$ is the capacity of channel. $(T-\tau)$ is the time left to transmit data after sensing duration.

The above definition of throughput is actually the available channel resource, not the exact throughput obtained by SU.

In order to study the actual optimal sensing order in practical systems, as in Figure 1, we use the actual throughput of SU's data traffic defined by the actually transmitted data in the actual time spent:

$$
\begin{aligned}
R_{2} & =\frac{L(\text { transmitted data }) \text { bit }}{t(\text { total time spend }) \mathrm{s}} \\
& = \begin{cases}\frac{L}{\tau+L / C}, & L<(T-\tau) C, \\
\frac{T-\tau}{T} C, & L \geq(T-\tau) C .\end{cases}
\end{aligned}
$$

This value is the actual throughput for the data traffic $L$. In addition, $R_{1}=R_{2}$ when $L \geq(T-\tau) C$.

\section{Performance Analysis}

3.1. Energy Detection. We adopt energy detection in the sensing duration as in [3]. Local spectrum sensing problem using energy detection can be formulated as the following binary hypothesis test:

$$
\begin{gathered}
H_{1}: y(n)=h(n) s(n)+u(n), \\
H_{0}: y(n)=u(n)
\end{gathered}
$$

where $H_{0}$ and $H_{1}$ denote that the PU on the channel is absent and present, respectively. $y(n)$ is the received signal at the $n$th sample in SU. The noise $u(n)$ is a Gaussian, independent, and identically distributed (i.i.d) random process with mean zero and variance $\sigma_{u}^{2}$. The primary signal $s(n)$ is an i.i.d random process with mean zero and variance $\sigma_{s}^{2}$. We assume that the primary signal $s(n)$ is complex PSK modulated and the noise $u(n)$ is circularly symmetric complex Gaussian (CSCG) signal. $h(n)$ denotes the channel gain of the listening channel between the PU and SU. Similar with the analysis in [3], we assume that the sensing time is smaller than the coherence time of the channel, so $h(n)$ can be viewed as time invariant during the sensing process, $h(n)=h$. We denote by $\gamma=$ $|h|^{2} \sigma_{s}^{2} / \sigma_{u}^{2}$ the signal-to-noise ratio (SNR) at the SU when PU exists. 

by

Following the analysis in [3], we use the test statistic given

$$
Y=\frac{1}{N} \sum_{n=1}^{N}|y(n)|^{2}
$$

where $N$ denotes the number of samples. Using central limit theorem (CLT), for a large $N$, the probability density function (PDF) of $Y$ under hypothesis $H_{0}$ can be approximated by a Gaussian distribution with mean $u_{0}=\sigma_{u}^{2}$ and variance $\sigma_{0}^{2}=(1 / N) \sigma_{u}^{4}$. The PDF of $Y$ under hypothesis $H_{1}$ can be approximated by a Gaussian distribution with mean $u_{1}=$ $(\gamma+1) \sigma_{u}^{2}$ and variance $\sigma_{1}^{2}=(1 / N)(2 \gamma+1) \sigma_{u}^{4}$ as

$$
\begin{gathered}
H_{0}: Y(i) \sim N\left(\sigma_{u}^{2}, \frac{1}{N} \sigma_{u}^{4}\right), \\
H_{1}: Y(i) \sim N\left((\gamma+1) \sigma_{u}^{2}, \frac{1}{N}(2 \gamma+1) \sigma_{u}^{4}\right) .
\end{gathered}
$$

When the detection threshold is $\lambda$, the probability of false alarm is given by

$$
P_{f}=\operatorname{Pr}\left[Y>\lambda \mid H_{0}\right]=Q\left(\frac{\lambda-\sigma_{u}^{2}}{\sigma_{u}^{2}} \sqrt{N}\right) .
$$

The probability of detection is given by

$$
P_{d}=\operatorname{Pr}\left[Y>\lambda \mid H_{1}\right]=Q\left(\left(\frac{\lambda}{\sigma_{u}^{2}}-\gamma-1\right) \sqrt{\frac{\tau f_{s}}{2 \gamma+1}}\right),
$$

where

$$
Q(x)=\frac{1}{\sqrt{2 \pi}} \int_{x}^{\infty} \exp \left(-\frac{t^{2}}{2}\right) d t .
$$

We denote sampling frequency by $f_{s}$; when the sensing time is $\tau$, the number of samples will be $N=\tau f_{s}$. Combining (6) and (7), the probability of false alarm is given by

$$
P_{f}=Q\left(\sqrt{2 \gamma+1} Q^{-1}\left(P_{d}\right)+\sqrt{\tau f_{s}} \gamma\right)
$$

3.2. Sensing-Throughput Tradeoff. We focus on the throughput of SU when PU is inactive, as in [2]; then $C$ denotes the channel capacity when it operates in the absence of primary users. We denote by $P_{0}=P\left(H_{0}\right)$ the probability for which the primary user is inactive; then the throughput of SU is given by

$$
R= \begin{cases}P_{0}\left(1-P_{f}\right) \frac{L}{\tau+L / C}, & L<(T-\tau) C, \\ P_{0}\left(1-P_{f}\right) \frac{T-\tau}{T} C, & L \geq(T-\tau) C .\end{cases}
$$

We focus on the first situation, that is, $L<(T-\tau) C$, because this one can show the effect of data traffic, and the other situation $(L \geq(T-\tau) C)$ is the traditional study [2].

There is a tradeoff between sensing and transmission. When $P_{d}$ is fixed to provide enough protection for PU, $P_{f}$ is a decreasing function of sensing time $\tau$ since $Q(x)$ is a monotonically decreasing function of $x$. When $\tau$ is longer, $\left(1-P_{f}\right)$ increases, but the value of $L /(\tau+L / C)$ in (10) decreases at the same time.

The objective is to find the optimal sensing time $\tau^{*}$ for SU's traffic $L$ to maximize the throughput while PU is sufficiently protected. The optimization problem can be stated as

$$
\begin{aligned}
\max _{\tau} \quad R= & P_{0}\left(1-P_{f}\right) \frac{L}{\tau+L / C} \\
= & P_{0}\left(1-Q\left(\sqrt{2 \gamma+1} Q^{-1}\left(P_{d}\right)+\sqrt{\tau f_{s}} \gamma\right)\right) \\
& \times \frac{L}{\tau+L / C}
\end{aligned}
$$

subject to $P_{d} \geq \overline{P_{d}}$,

where $\overline{P_{d}}$ is the target probability of detection. For example, $\overline{P_{d}}=0.9$ in IEEE802.22 WRAN [9].

3.3. Optimal Sensing Time. In problem (11), $Q(x)$ is a monotonically decreasing function of $x, Q^{-1}(x)$ is a monotonically decreasing function of $x$, and then $R$ is a monotonically decreasing function of $P_{d}$. Therefore, the optimal solution is achieved with equality constraint in (11). So the optimization problem can be transferred into

$$
\begin{aligned}
\max _{\tau} R & =P_{0}\left(1-P_{f}\left(\tau, \overline{P_{d}}\right)\right) \frac{L}{\tau+L / C} \\
& =P_{0}\left(1-Q\left(\sqrt{2 \gamma+1} Q^{-1}\left(\overline{P_{d}}\right)+\sqrt{\tau f_{s}} \gamma\right)\right) \frac{L}{\tau+L / C} .
\end{aligned}
$$

Theorem 1. There exists only one optimal sensing time $\tau^{*}$ which maximizes the throughput $R$ when $P_{f} \leq 0.5\left(P_{f} \leq 0.5\right.$ is a typical value range in cognitive radio).

Proof. From (12), we have

$$
R^{\prime}(\tau)=P_{0} L \frac{P_{f}(\tau)-(\tau+L / C) P_{f}^{\prime}(\tau)-1}{(\tau+L / C)^{2}},
$$

and then

$$
\begin{aligned}
R^{\prime \prime}(\tau)=\frac{P_{0} L}{\left(\tau+\frac{L}{C}\right)^{3}}( & -\left(\tau+\frac{L}{C}\right)^{2} P_{f}^{\prime \prime}(\tau)-2 P_{f}(\tau) \\
& \left.+2\left(\tau+\frac{L}{C}\right) P_{f}^{\prime}(\tau)-2\right) .
\end{aligned}
$$

According to (9),

$$
\begin{aligned}
P_{f}^{\prime}(\tau)= & -\frac{\gamma \sqrt{f_{s}}}{2 \sqrt{2 \pi \tau}} \\
& \times \exp \left(-\frac{\left(\sqrt{2 \gamma+1} Q^{-1}\left(\overline{P_{d}}\right)+\sqrt{\tau f_{s}} \gamma\right)^{2}}{2}\right)<0 .
\end{aligned}
$$


When $P_{f} \leq 0.5, P_{f}^{\prime}(\tau)$ is monotonically increasing function of $\tau$ [3], which means $P_{f}^{\prime \prime}(\tau)>0$.

Applying $P_{f}>0, P_{f}^{\prime}(\tau)<0$, and $P_{f}^{\prime \prime}(\tau)>0$ to (14), we can get $R^{\prime \prime}(\tau)<0$, which means that $R^{\prime}(\tau)$ is a monotonically decreasing function of $\tau$.

In addition, according to (9) and (15), $\lim _{\tau \rightarrow 0} P_{f}(\tau)=1$, and $\lim _{\tau \rightarrow 0} P_{f}^{\prime}(\tau)=-\infty$, then

$$
\begin{gathered}
\lim _{\tau \rightarrow 0} R^{\prime}(\tau)=-P_{0} L \frac{\lim _{\tau \rightarrow 0} P_{f}^{\prime}(\tau)}{L / C}=+\infty \\
\lim _{\tau \rightarrow(T-L / C)} R^{\prime}(\tau)=P_{0} L \frac{P_{f}(T-L / C)-T P_{f}^{\prime}(T-L / C)-1}{T^{2}}
\end{gathered}
$$

Since $R(\tau)>0, \lim _{\tau \rightarrow 0} R^{\prime}(\tau)=+\infty, R^{\prime \prime}(\tau)<0, R(\tau)$ is concave for the range of $\tau$ when $P_{f} \leq 0.5$. We can have the following conclusions.

(1) If $\lim _{\tau \rightarrow(T-L / C)} R^{\prime}(\tau)<0$, that is, $P_{f}(T-L / C)-$ $T P_{f}^{\prime}(T-L / C)-1<0$, there is one optimal sensing time $\tau^{*}$ which maximizes the throughput $R$.

(2) If $\lim _{\tau \rightarrow(T-L / C)} R^{\prime}(\tau) \geq 0$, that is, $P_{f}(T-L / C)-$ $T P_{f}^{\prime}(T-L / C)-1 \geq 0$, the optimal sensing time $\tau^{*}$ is $\tau^{*}=T-$ $L / C$.

This result means that condition $L \geq(T-\tau) C$ is meted (10), and this situation has been studied in [2], where $R(\tau)$ is concave for the range of $\tau$ when $P_{f} \leq 0.5$; there is also one optimal sensing time (denoted by $\tau_{1}^{*}$ ) which maximizes the throughput $R$.

Combining our research and study in [2], the conclusion is as follows.

$$
\begin{aligned}
& \text { If } \tau_{1}^{*} \leq T-L / C \text {, the optimal sensing time is } \tau^{*}= \\
& T-L / C \text {. } \\
& \text { Else, } \tau^{*}=\tau_{1}^{*} .
\end{aligned}
$$

(3) In particular, according to (13), when $T \rightarrow+\infty$, $\lim _{\tau \rightarrow(T-L / C)} R^{\prime}(\tau)<0$, which means that when there is no limitation of time slot, $R(\tau)$ is concave for the range of $\tau$ when $P_{f} \leq 0.5$, there is only one optimal sensing time $\tau^{*}$ which maximizes the throughput $R$.

In all, there is only one optimal sensing time $\tau^{*}$ which maximizes the throughput $R$ when $P_{f} \leq 0.5$. So efficient search algorithms can be developed to find the optimal sensing time.

In addition, the optimal sensing time in our model is related to the traffic $L$ and the channel capacity $C$, which is different from research in [2].

\section{Numerical Results and Discussions}

In this section, we present the simulation results to evaluate the performance of the proposed method. Without loss of generality, similar with [2], the following parameters are set.
The bandwidth of PU channel is $6 \mathrm{MHz}$, the SNR of SU's signal without PU's single is $0 \mathrm{~dB}$, and the sampling frequency is the same as the bandwidth. The probability of channel idle is $P_{0}=0.8$, and the target probability of detection is $\overline{P_{d}}=0.9$. We are also interested in low SNR regime as in [2].

We assume that the model of data traffic of SU is constant bit rate (CBR) traffic [10], which is a popular traffic source in network simulation. The data rate is set as $\lambda=[16,64,256$, $384,512,768,1024,1280,1536,1792] \mathrm{Kbit} / \mathrm{s}$; then the data amount for transmission in next slot is $L=\lambda T$. These data rates include some typical voice and video formats, such as G.728LD-CELP (16 Kbit/s), G.711PCM (64 Kbit/s), WMV (256 Kbit/s), AVI/RM (384 Kbit/s), flash (512 Kbit/s), and $\mathrm{H} 264$ (768 Kbit/s), to simulate the traffic generated by various applications.

Figures 2 and 3 show that the optimal sensing time obtained by our method is different with the value obtained by the method in [2] (capacity based [2]). Note that the throughput in the top figure (capacity based [2]) is the throughput defined by (1), not the real throughput for data traffic defined by (2). In addition, the optimal sensing time in [2] is different when the frame time $T$ is different, but in our method, the optimal sensing time is only determined by SU's traffic.

Figures 2, 4, and 5 show that the optimal sensing time is different when the SNR is different, and the value in [2] and in our method is also different. Importantly, there is only one optimal sensing time value in different SNR values, which proves our analysis.

Figures 6, 7, 8, and 9 show that the optimal sensing time varies with the input data traffic of SU in the proposed method. However, with fixed frame time $T$ and $S N R$ value, the optimal sensing time obtained by [2] is a fixed value, which is not related with traffic.

The results show that the real throughput for traffic obtained by our method is obviously better than the method in [2]. For example, in Figure 6, with some typical applications, 157 percent improvement for G.711PCM (64 Kbit/s), 114 improvement for WMV (256 Kbit/s) and 109 improvement for AVI/RM (384 Kbit/s), because the sensing time is optimized by considering the traffic.

When the frame time and SNR value are different, the optimal sensing time and the optimal throughput change. Importantly, the performance of the proposed method is always better than the method in [2], which fails to take the SU's traffic into consideration.

In addition, when the traffic is heavy, the data queue may be almost full; the throughput becomes almost the same, which verifies that the model in [2] is a special case of our model.

\section{Conclusions}

In this paper, we investigate the Sensing-Throughput Tradeoff problem in CRN, considering the effect of traffic of SU. We reformulated this problem by removing the assumption that the SUs always have countless data packets to transmit. The proposed model is more suitable for practical systems and 


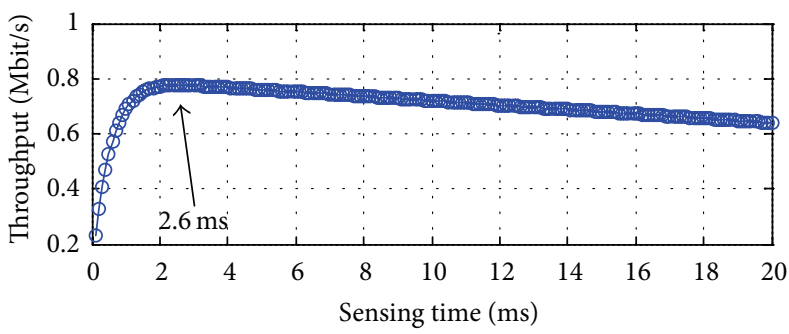

$\rightarrow$ Capacity based [2]

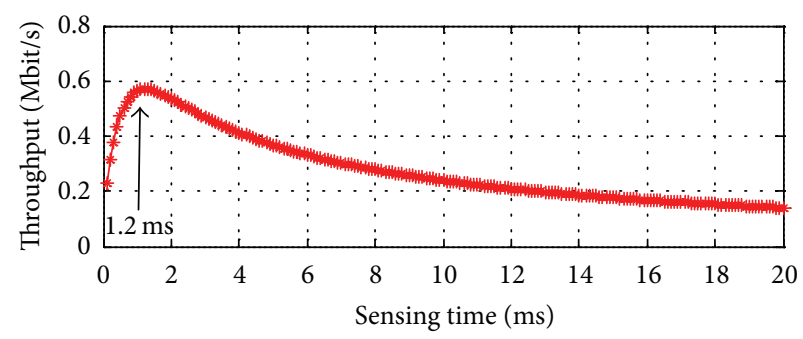

$\rightarrow$ Proposed traffic based

(a)

(b)

FIgURE 2: The optimal sensing time, $\lambda=256 \mathrm{Kbit} / \mathrm{s}, T=100 \mathrm{~ms}$, and SNR $=-15 \mathrm{~dB}$.

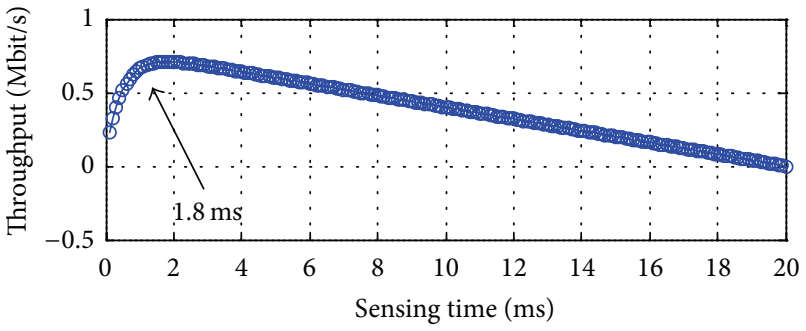

$\rightarrow$ Capacity based $[2]$

(a)

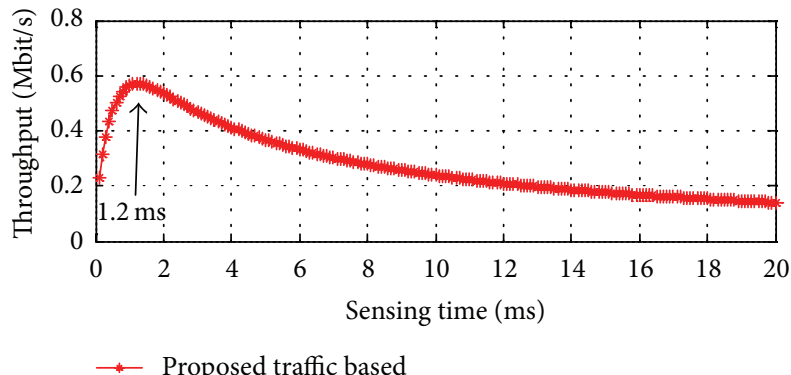

(b)

Figure 3: The optimal sensing time, $\lambda=256 \mathrm{Kbit} / \mathrm{s}, T=20 \mathrm{~ms}$, and SNR $=-15 \mathrm{~dB}$.

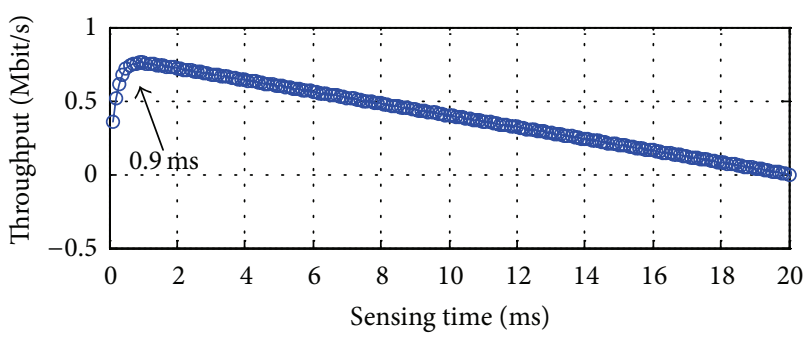

$\multimap$ Capacity based [2]

(a)

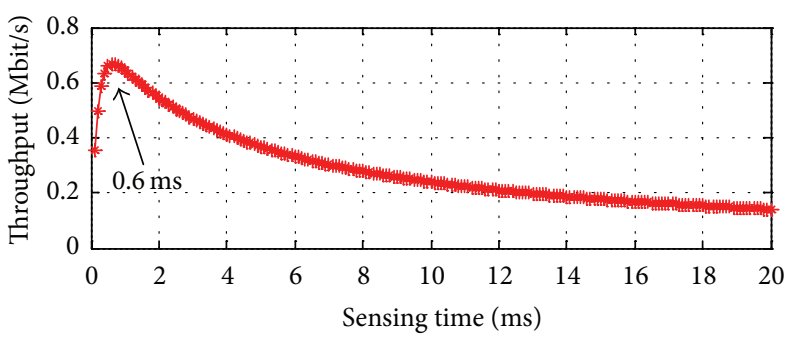

$\rightarrow$ Proposed traffic based

(b)

Figure 4: The optimal sensing time, $\lambda=256 \mathrm{Kbit} / \mathrm{s}, T=100 \mathrm{~ms}$, and $\mathrm{SNR}=-13 \mathrm{~dB}$.

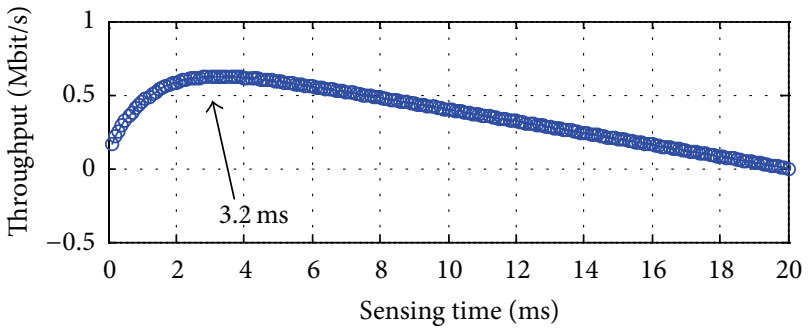

$\rightarrow$ Capacity based [2]

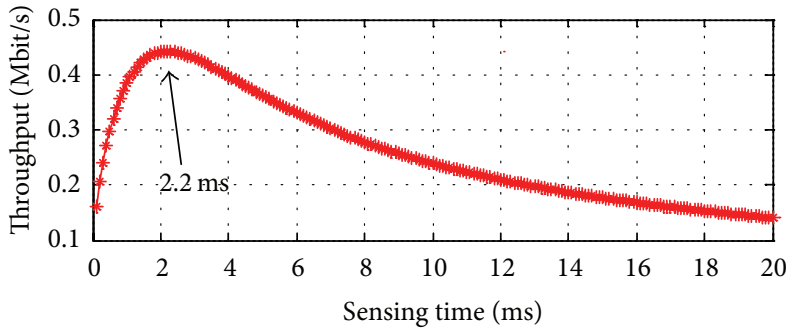

$\rightarrow$ Proposed traffic based

(b)

FIgUre 5: The optimal sensing time, $\lambda=256 \mathrm{Kbit} / \mathrm{s}, T=100 \mathrm{~ms}$, and SNR $=-17 \mathrm{~dB}$. 


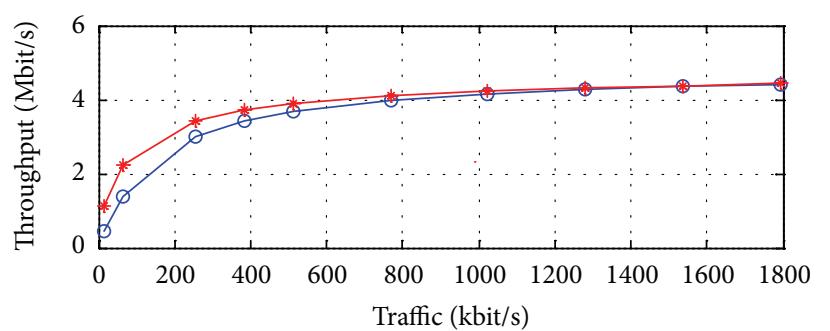

$\rightarrow$ Capacity based [2]

$\rightarrow-$ Proposed traffic based

(a)

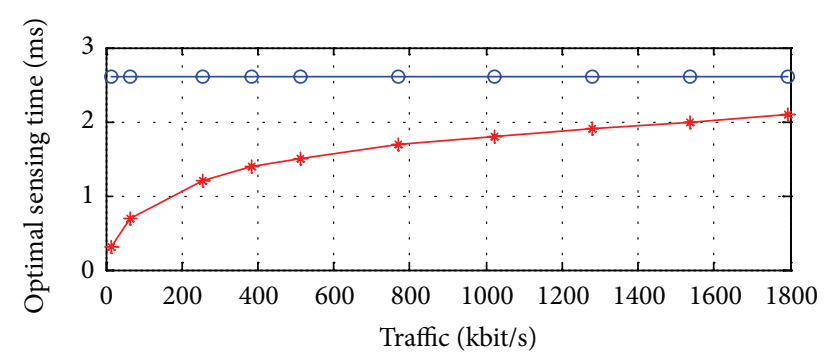

(b)

FIgURE 6: The traffic versus throughput, $T=100 \mathrm{~ms}$ and $\mathrm{SNR}=-15 \mathrm{~dB}$.

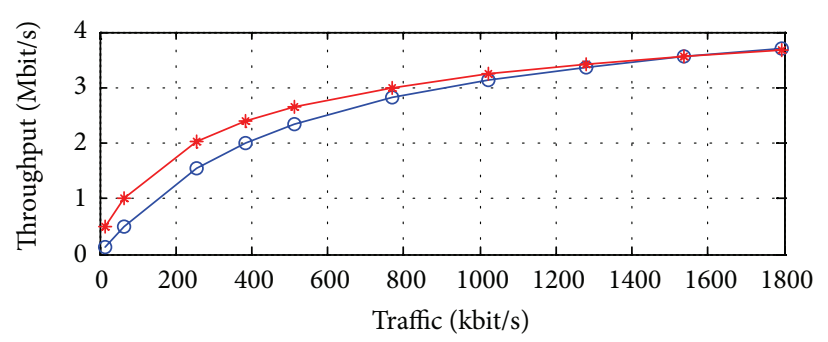

- Capacity based [2]

$\rightarrow-$ Proposed traffic based

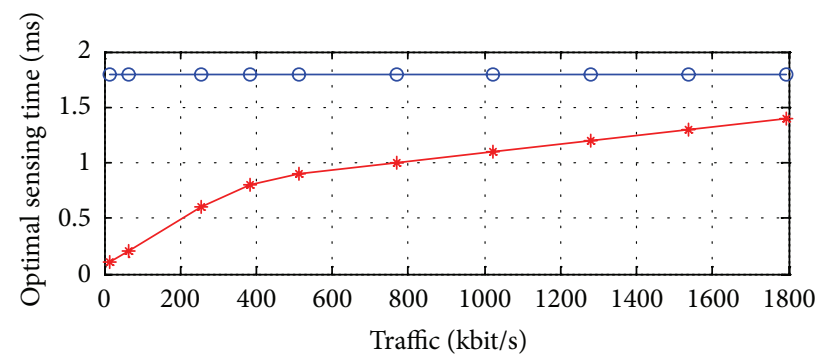

(b)

FIgURE 7: The traffic versus throughput, $T=20 \mathrm{~ms}$ and $\mathrm{SNR}=-15 \mathrm{~dB}$.

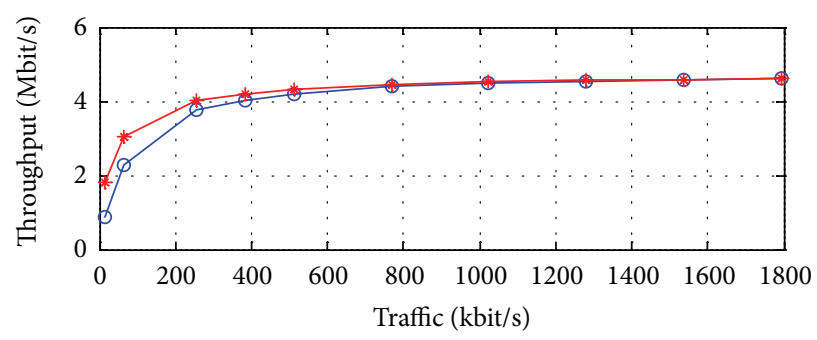

$\rightarrow$ Capacity based [2]

$\rightarrow$ Proposed traffic based

(a)

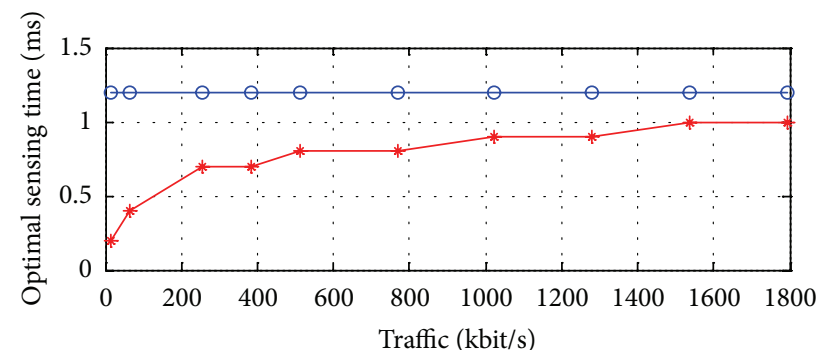

(b)

FIgURE 8: The traffic versus throughput, $T=100 \mathrm{~ms}$ and $\mathrm{SNR}=-13 \mathrm{~dB}$.

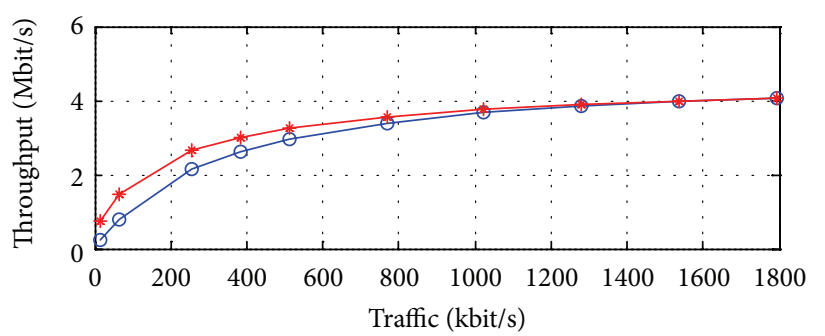

- Capacity based [2]

$\rightarrow$ Proposed traffic based

(a)

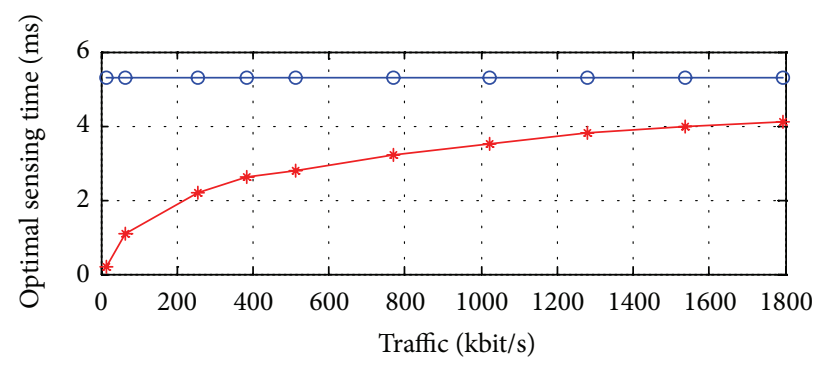

(b)

FIgURE 9: The traffic versus throughput, $T=100 \mathrm{~ms}$ and SNR $=-17 \mathrm{~dB}$. 
can be regarded as a more general model compared with the existing work, because the traditional Sensing-Throughput Tradeoff model can be seen as a special case of our model. We also prove that the throughput is a concave function of sensing time when the probability of false alarm is less than 0.5 , and there is only one optimal sensing time value which is determined by the data traffic. Simulation results show that the proposed method achieves better performance than the existing approaches.

\section{Conflict of Interests}

The authors declare that there is no conflict of interests regarding the publication of this paper.

\section{Acknowledgments}

This work was supported by the National Natural Science Foundation of China (Grant nos. 61172062 and 61301160), by Jiangsu Province Natural Science Foundation (Grant no. BK2011116), and in part by the National Basic Research Program of China (Grant no. 2009CB320400). This work was also supported by the National Science Foundation of China under Grant no. 61401508.

\section{References}

[1] J. Mitola III and G. Q. Maguire Jr., "Cognitive radio: making software radios more personal," IEEE Personal Communications, vol. 6, no. 4, pp. 13-18, 1999.

[2] Y.-C. Liang, Y. Zeng, E. C. Y. Peh, and A. T. Hoang, "Sensingthroughput tradeoff for cognitive radio networks," IEEE Transactions on Wireless Communications, vol. 7, no. 4, pp. 1326-1337, 2008.

[3] E. C. Y. Peh, Y.-C. Liang, Y. L. Guan, and Y. Zeng, “Optimization of cooperative sensing in cognitive radio networks: a sensingthroughput tradeoff view," IEEE Transactions on Vehicular Technology, vol. 58, no. 9, pp. 5294-5299, 2009.

[4] S. Stotas and A. Nallanathan, "Overcoming the sensingthroughput tradeoff in cognitive radio networks," in Proceedings of the IEEE International Conference on Communications (ICC '10), May 2010.

[5] S. Althunibat, M. Di Renzo, and F. Granelli, "Cooperative spectrum sensing for cognitive radio networks under limited time constraints," Computer Communications, vol. 43, pp. 5563, 2014.

[6] L. Tang, Y. Chen, E. L. Hines, and M.-S. Alouini, "Effect of primary user traffic on sensing-throughput tradeoff for cognitive radios," IEEE Transactions on Wireless Communications, vol. 10, no. 4, pp. 1063-1068, 2011.

[7] Y.-J. Choi, Y. Xin, and S. Rangarajan, "Overhead-throughput tradeoff in cooperative cognitive radio networks," in Proceedings of the IEEE Wireless Communications and Networking Conference (WCNC '09), pp. 1-9, Budapest, Hungary, April 2009.

[8] S. Althunibat, R. Palacios, and F. Granelli, "Performance optimisation of soft and hard spectrum sensing schemes in cognitive radio," IEEE Communications Letters, vol. 16, no. 7, pp. 998-1001, 2012.

[9] IEEE 802.22 Wireless RAN, "Functional requirements for the 802.22 WRAN standard,” IEEE 802.22-05/0007r46, 2005.
[10] T. Karagiannis, M. Molle, M. Faloutsos, and A. Broido, "A nonstationary poisson view of internet traffic," in Proceedings of the 23rd Annual Joint Conference of the IEEE Computer and Communications Societies (INFOCOM '04), vol. 3, pp. 15581569, Hong Kong, March 2004. 


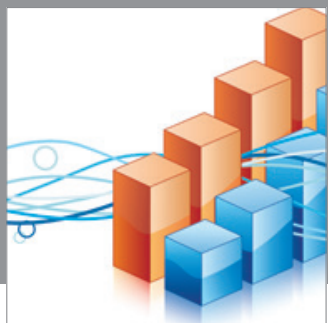

Advances in

Operations Research

mansans

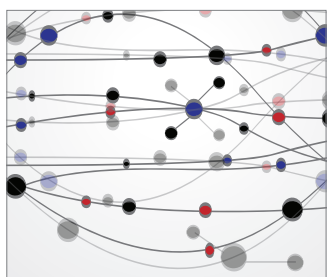

The Scientific World Journal
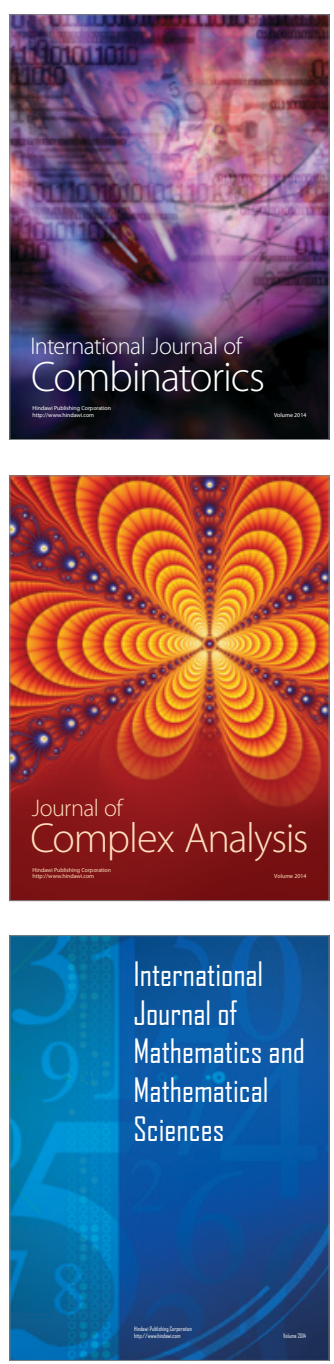
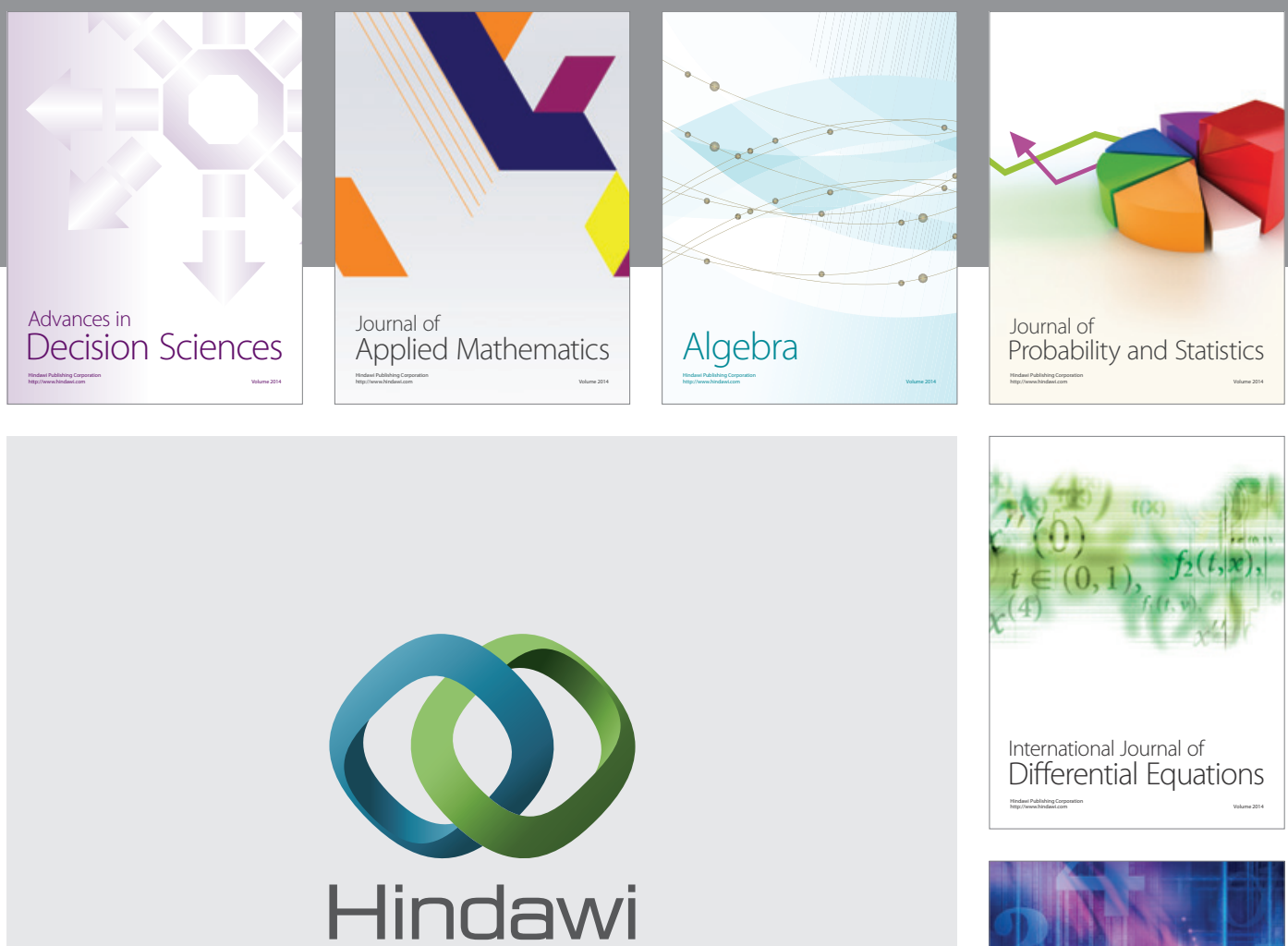

Submit your manuscripts at http://www.hindawi.com
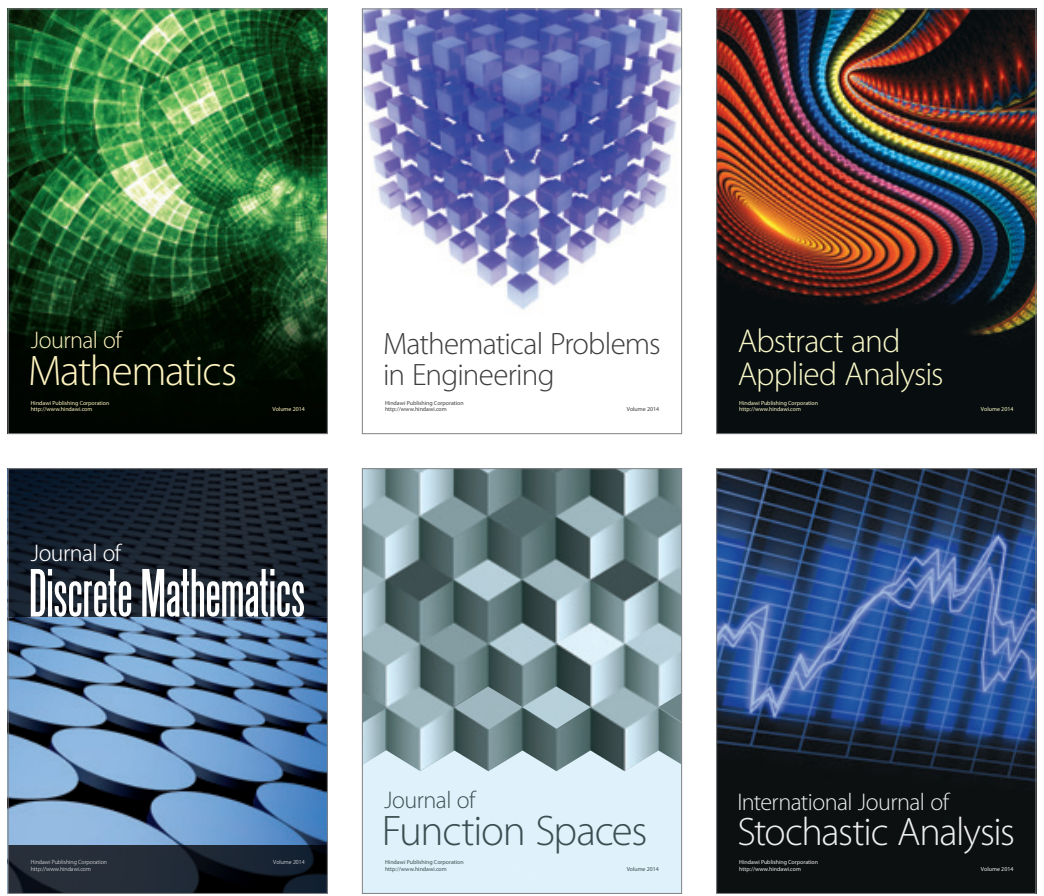

Journal of

Function Spaces

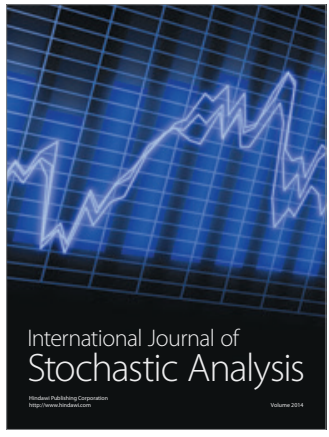

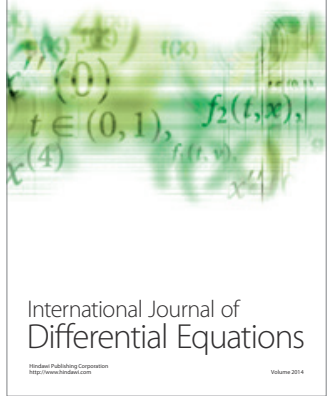
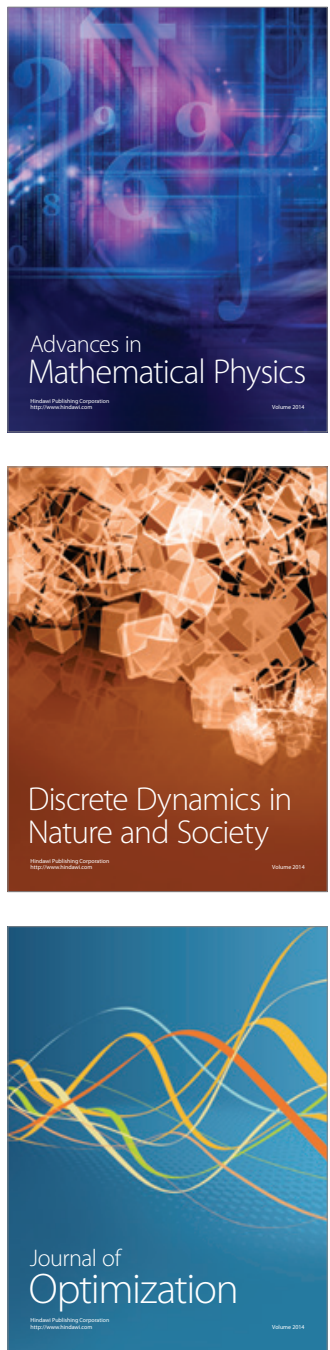